DOI: 10.1136/annrheumdis-2017-eular.3833

\section{SAT0221 SIX-MONTH PROTEINURIA MEASUREMENT PREDICTS RENAL RESPONSE AT 18 MONTHS IN LUPUS NEPHRITIS: ANALYSIS OF TWO PHASE III RANDOMIZED CLINICAL TRIALS}

M.D. Cascino ${ }^{1,2}$, T. Schindler ${ }^{3}$, L.M. Gomez Mendez ${ }^{2}$, P. Brunetta ${ }^{1}$ L. Dragone ${ }^{1}$, M. Dall'Era ${ }^{2}$, J. Garg ${ }^{1} .{ }^{1}$ Genentech, Inc., South San Francisco; ${ }^{2}$ University of California - San Francisco, San Francisco, United States; ${ }^{3} \mathrm{~F}$. Hoffmann-La Roche, Basel, Switzerland

Background: Early identification of patients with lupus nephritis (LN) likely to achieve complete renal response (CRR) may expedite the evaluation of new therapies and guide clinical care. Prior analyses have shown that early improvement in proteinuria is associated with subsequent renal response. ${ }^{1,2}$ Several ongoing LN trials including NOBILITY, an assessment of the efficacy of the anti-CD20 monoclonal antibody obinutuzumab in combination with standard of care immunosuppression, will evaluate proteinuric response at 6 months as a key secondary endpoint. ${ }^{3}$ Whether short-term response accurately predicts future CRR, however, is uncertain.

Objectives: To assess the predictive value of early measurements of the level of proteinuria and to identify proteinuria cutoffs that best identify patients who will achieve CRR at 18 months.

Methods: LUNAR and BELONG were multicenter, double-blinded studies that in total randomized 522 patients with ISN/RPS class III or class IV LN to blinded investigational infusions or placebo in combination with standard of care immunosuppression. ${ }^{4,5}$ CRR was assessed at 18 months and defined for this analysis as achievement of urine protein to creatinine ratio (UPCR) $<0.5$ with normal serum creatinine that was not increased from baseline by $>25 \%$. Bootstrapping was used to generate nonparametric receiver operating characteristic (ROC) curves and estimate area under the curve (AUC). The Youden index was used to identify UPCR cutoff values that maximize sensitivity and specificity. Positive predictive value (PPV) and negative predictive value (NPV) were calculated.

Results: ROC curves were constructed for proteinuria measurements at baseline and $3,6,9$, and 12 months after randomization (Figure 1). AUC increased from baseline to month $3(0.64$ vs. $0.80, P<0.001)$ and from month 3 to month 6 (0.80 vs. $0.84, P<0.01)$ but did not increase beyond month $6(P>0.05$ for each pairwise comparison). Achievement of 6 -month UPCR $<1$ was $83.8 \%$ sensitive and $71.0 \%$ specific for CRR at 18 months and had PPV and NPV of $64.9 \%$ and $87.2 \%$, respectively. Evaluation of lower 6 -month UPCR cutoff values yielded improvements in specificity and PPV but marked decreases in sensitivity and NPV. In multivariate analysis, the addition of 6-month serum creatinine and percent change in UPCR from baseline did not result in meaningful increases in AUC compared with 6-month proteinuria measurement alone.

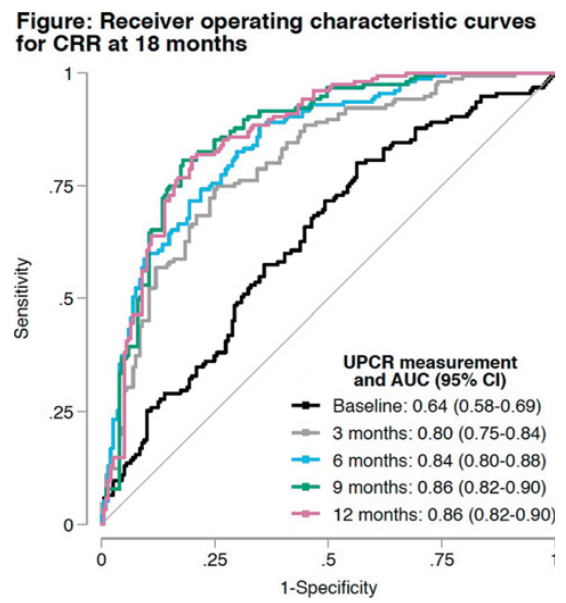

Conclusions: Level of proteinuria at 6 months alone was predictive of CRR at 18 months in aggregated data from two phase III LN clinical trials. After 6 months of treatment, UPCR $<1$ had high sensitivity and NPV for CRR at 18 months. This cutoff might be used to prospectively identify patients who are unlikely to achieve complete response within 18 months on the initial therapy for LN. The impact of these findings on guiding treatment decisions outside the setting of randomized clinical trials requires further investigation.

References:

[1] Tamirou Lupus Sci \& Med 2015.

[2] Dall'Era Arthritis Rheumatol 2015

[3] Schindler Ann Rheum Dis 2016.

[4] Rovin B Arthritis Rheumatol 2012.

[5] Mysler E Arthritis Rheumatol 2013.

[1] Trial registry numbers: NCT00282347 (LUNAR) and NCT00626197 (BELONG).

Disclosure of Interest: M. Cascino Employee of: Roche/Genentech, T. Schindler
Employee of: Roche/Genentech, L. Gomez Mendez Grant/research support from: Roche/Genentech, P. Brunetta Employee of: Roche/Genentech, L. Dragone Employee of: Roche/Genentech, M. Dall'Era: None declared, J. Garg Employee of: Roche/Genentech

DOI: 10.1136/annrheumdis-2017-eular.5414

\section{SAT0222 BIIB059, A MONOCLONAL ANTIBODY TARGETING BDCA2, SHOWS EVIDENCE OF BIOLOGICAL ACTIVITY AND EARLY CLINICAL PROOF OF CONCEPT IN SUBJECTS WITH ACTIVE CUTANEOUS LE}

$\underline{\text { R. Furie }}^{1}$, V.P. Werth ${ }^{2}$, J.F. Merola ${ }^{3}$, T.L. Reynolds ${ }^{4}$, L. Stevenson ${ }^{4}$, W. Wang ${ }^{4}$ K. Smirnakis ${ }^{4}$, C. Barbey ${ }^{5}$, C. Musselli ${ }^{4,4}$, B. Werneburg ${ }^{4}$, D. Rabah ${ }^{4}$,

N. Franchimont ${ }^{4} .{ }^{1}$ Northwell Health, Great Neck; ${ }^{2}$ Hospital of the University of Pennsylvania and the Veteran's Administration Medical Center, Philadelphia ${ }^{3}$ Brigham and Women's Hospital, Harvard Medical School, Boston; ${ }^{4}$ Biogen, Cambridge, United States; ${ }^{5}$ Biogen, Zug, Switzerland

Background: Type I interferons (IFN-I) are central to the pathogenesis of systemic lupus erythematosus (SLE). BDCA2 is a plasmacytoid dendritic cell (pDC)-specific receptor that, upon engagement, inhibits the production of IFN-I and other inflammatory mediators. Targeting BDCA2, therefore, represents an attractive therapeutic strategy for inhibiting pDC-driven inflammation that is such a key feature of SLE pathogenesis. BIIB059, an investigational anti-BDCA2 humanized monoclonal antibody, has been shown to engage BDCA2, and this interaction leads to BDCA2 internalization and the consequent in vitro inhibition of TLR-induced IFN-I production by pDCs (Pellerin 2015).

Objectives: This first-in-patient study aimed to assess safety, tolerability, pharmacokinetic (PK) and pharmacodynamic (PD) effects and clinical activity of BIIB059 in adult SLE patients with active cutaneous lupus (CLE) following administration of a single BlIB059 dose.

Methods: A Phase $1 \mathrm{~b}$ randomized, double-blinded, placebo controlled, multicenter clinical trial was conducted in 12 adult SLE subjects (meeting 1997 ACR criteria) with active cutaneous manifestations (including acute, sub-acute and/or chronic cutaneous forms of cutaneous lupus erythematosus (CLE)). Subjects received a single IV administration of either BIIB059 $20 \mathrm{mg} / \mathrm{kg}(n=8)$ or placebo $(n=4)$. A panel of IFN-responsive genes (IRG) was assessed from whole blood by qPCR at baseline and several post-dose time points. Skin biopsies from active lesions were obtained and evaluated at baseline and week 4 for IFN-regulated proteins, including MXA and IFITM3 using quantitative immunohistochemistry. CLE disease activity was assessed using the Cutaneous Lupus Erythematosus Disease Area and Severity Index (CLASI), and safety data, including adverse events (AEs) and laboratory tests, were also collected.

Results: Most SLE subjects had high IRG signatures in the blood. Skin biopsies demonstrated features of inflammation consistent with active CLE, including elevated expression of MxA and other IFN-regulated proteins. A single dose of BIIB059 decreased the expression of IRG in blood and MxA and IFITM3 proteins in the skin in most patients. CD45+ cells were reduced in skin biopsies of BIIB059-treated patients. The reduction in inflammatory cells as well as MxA and IFITM3 expression at week 4 correlated with improvement in CLASI activity score at multiple timepoints post-dose. BIIB059 was generally well tolerated with no discontinuations due to AEs. The incidence of AEs was similar between BIIB059and placebo-treated SLE subjects, and most AEs were mild or moderate in severity.

Conclusions: A single dose of BIIB059 resulted in inhibition of the IRG in peripheral blood and MxA and IFITM3 expression in lesional skin of SLE subjects, consistent with BIIB059's proposed mechanism of action. The clinical and biomarker data together confirm the role of human pDCs in the pathogenesis of SLE, and support further development of BIB059 in SLE.

Disclosure of Interest: R. Furie Consultant for: Biogen, V. Werth Grant/research support from: Biogen, Consultant for: Biogen, J. Merola Grant/research support from: Biogen, Consultant for: Biogen, AbbVie, Amgen, Eli Lilly, Novartis, Pfizer, Janssen, Mallinckrodt, Momenta, Speakers bureau: AbbVie, Eli Lilly, T. Reynolds Shareholder of: Biogen, Employee of: Biogen, L. Stevenson Shareholder of: Biogen, Employee of: Biogen, W. Wang Shareholder of: Biogen, Employee of: Biogen, K. Smirnakis Shareholder of: Biogen, Employee of: Biogen, C. Barbey Shareholder of: Biogen, Employee of: Biogen, C. Musselli Shareholder of: Biogen, Employee of: Biogen, B. Werneburg Shareholder of: Biogen, Employee of: Biogen, D. Rabah Shareholder of: Biogen, Employee of: Biogen, N. Franchimont Shareholder of: Biogen, Employee of: Biogen DOI: 10.1136/annrheumdis-2017-eular.6259

\section{SAT0223 INDIRECT COMPARATIVE CLINICAL EFFECTIVENESS OF INTRAVENOUS AND SUBCUTANEOUS FORMULATIONS OF BELIMUMAB FOR THE TREATMENT OF ADULT PATIENTS WITH ACTIVE, AUTOANTIBODY-POSITIVE SYSTEMIC LUPUS ERYTHEMATOSUS WITH HIGH DISEASE ACTIVITY}

D. Parks ${ }^{1}$, S. Ramachandran ${ }^{1}$, M. Kurtinecz ${ }^{1}$, Y. Asukai ${ }^{2}$

R. Alfonso-Cristancho ${ }^{1} .{ }^{1}$ GSK, Collegeville, United States; ${ }^{2}$ GSK, Uxbridge, United Kingdom

Background: The efficacy of belimumab (BEL) vs placebo (PBO), in adult 